\title{
Three-Dimensional Measurement of a Remote Object with a Single CCD camera
}

\author{
CHENG-CHUAN CHEN ${ }^{*}$, CHEN-CHIEN HSU ${ }^{\S}$, TI-HO WANG ${ }^{*}$, CHUN-WEI HUANG ${ }^{\S}$ \\ *Department of Electrical Engineering, St. John's University \\ 499 Tam King Rd., Sec. 4, Tam-Sui, Taipei County, 25135
}

TAIWAN

${ }^{\S}$ Department of Electrical Engineering, Tamkang University

151 Ying-chuan Rd., Tam-Sui, Taipei County, 25137

TAIWAN

\#Department of Electronic Engineering, St. John's University

499 Tam King Rd., Sec. 4, Tam-Sui, Taipei County, 25135

TAIWAN

\begin{abstract}
This paper presents a novel method by regulating the height of a CCD camera to measure the width and height of a targeted object. That is, three-dimensional measurement can be obtained with the use of a single CCD camera. To facilitate the measurement using various kinds of CCD cameras, parameters pertinent to the cameras can be established via a proposed mechanism in this paper. As a result, any kinds of CCD cameras can be used to perform three dimensional measurement of a targeted object via software implementation of the proposed measuring method. No changes to the circuits or structure of the camera are required.
\end{abstract}

Keywords: - CCD camera, distance measurement, three-dimensional measurement.

\section{Introduction}

There are generally two ways for measuring distance: contact and non-contact approaches. For non-contact measurement, various solutions can be used, like ultrasonic distance measurement [1]-[2], laser reflection methods [3]-[4]. Because these two methods use the theory of reflection, object reflectivity plays an import role. If the reflection surface is undesired, the measuring system generally performs poorly or not at all. On the other hand, image-based measuring systems based on pattern recognition or image analysis techniques [5]-[6] generally demand huge amount of storage capacity and high-speed DSP processors, which inevitably imposes a critical constraint for practical applications. To overcome the problems and difficulties encountered by the existing techniques, an image-based measuring system without complex calculation entitled Image-Based Distance Measuring System (IBDMS) is presented in this paper.

The configuration of the proposed IBDMS [7]-[9] is very simple, consisting of only a single CCD camera and two parallel laser projectors beside the camera. Because the disposition of the laser projectors and the optical origin of the CCD camera, which form a straight line, two laser-projected spots will appear on the same scan line in a CCD image. As a result, processing a single scan line, rather than the whole image, is only required to identify the projected spots in the CCD image. The methods of computing complex video signals of a whole image via either pattern recognition or image analysis are therefore no longer required. Based on an established relationship between the distance and pixel counts between the projected spots on a CCD image, 3-dimensional measurement of a distant object can be obtained via this proposed method. Note that the method proposed in this paper is quite different from those which use two CCD cameras [10]-[11] during the measuring. By regulating the height of a CCD camera, only one CCD camera is only required to overcome the problem of image loss [12]-[13]. Because the same parameters are used for taking pictures through out the measurements before and after the regulation of the CCD camera, thus we are able to increase the accuracy of the measurement. The image of the targeted object in images changes, when the CCD camera goes straight up or down. Therefore, by using a vertical scan line, we are able to know the image movement without the need to process the whole image. Knowing the variation of pixel counts in the images because of the 
camera movement, we can obtain three-dimensional measurement of the targeted object by using the proposed method. Practical experiments will be conducted in this paper to validate the effectiveness and viability of the proposed approach.

The paper is organized as follows. Section 2 introduces the distance measuring method. The proposed three-dimensional measurement is described in Section 3. The determination of intrinsic parameters for digital cameras is given in Section 4. Experiment results are presented in Section 5. Conclusions are drawn in Section 6.

\section{The Distance Measuring System}

Fig. 1 shows the diagram of the measuring system, where a shaft is used to control the height of the CCD camera along the vertical direction. The distance due to up and down of the camera can observed by the optical ruler. A particular distance of the movement can also be obtained via settings of switches $\mathrm{SW}_{1}$ and $\mathrm{SW}_{2}$. When the CCD camera moves along the vertical direction, the object in images will according move vertically as shown in Fig. 2, similar to the distance measuring systems [10]-[11] using the two CCD cameras. In this paper, a single CCD camera is only used, which circumvents the problems of measuring inaccuracy due to different camera parameters during the measurement. The convenience of the proposed approach where movement of the camera is directed along the vertical direction also contributes to the measurement accuracy. Other similar measuring systems include the triangular measuring method [7] and parallel measuring method [8]-[9]. These two methods, however, have to use two laser projectors for projecting laser beams onto the targeted object similar to an optical ruler, based on which measurement of distance can proceed. This fixture of the CCD camera together with the laser projectors is not easy to install. The projection of laser beams also requires extra electricity supply.

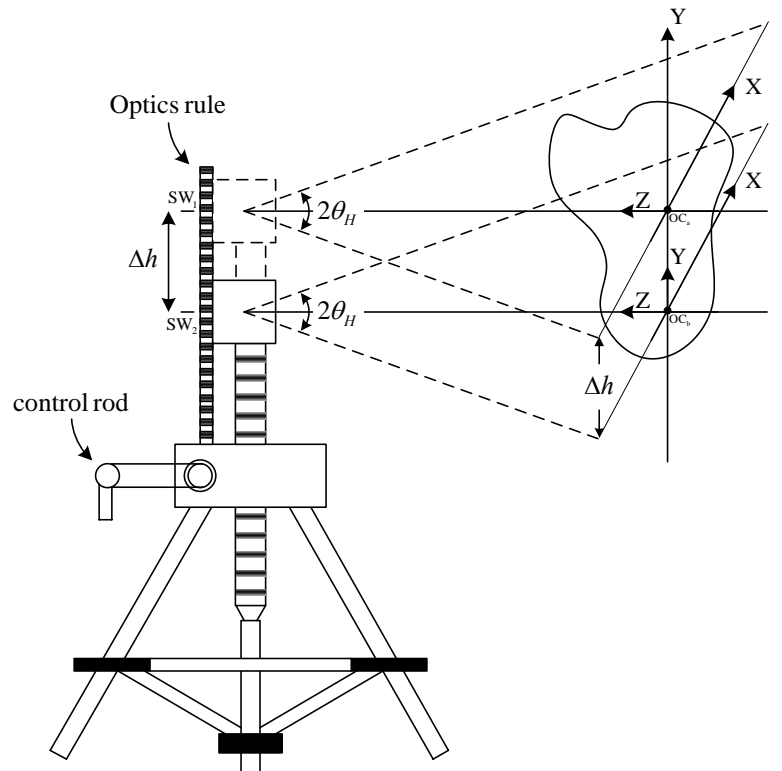

Fig.1 Diagram of the measuring system.

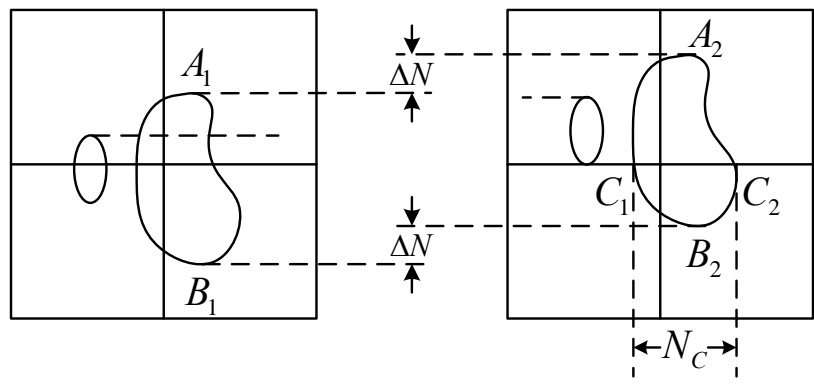

Fig. 2 Movement of the objects in images due to the movement of the CCD camera.

When the CCD camera is vertically moved for $\Delta h$, objects in the image will move as well in terms of pixel variation. As shown in Fig. 2, the movement of the object in the image is measured and represented as $\Delta N$ pixels. In what follows, we will describe the relationship between $\Delta h$ and $\Delta N$ in detail.

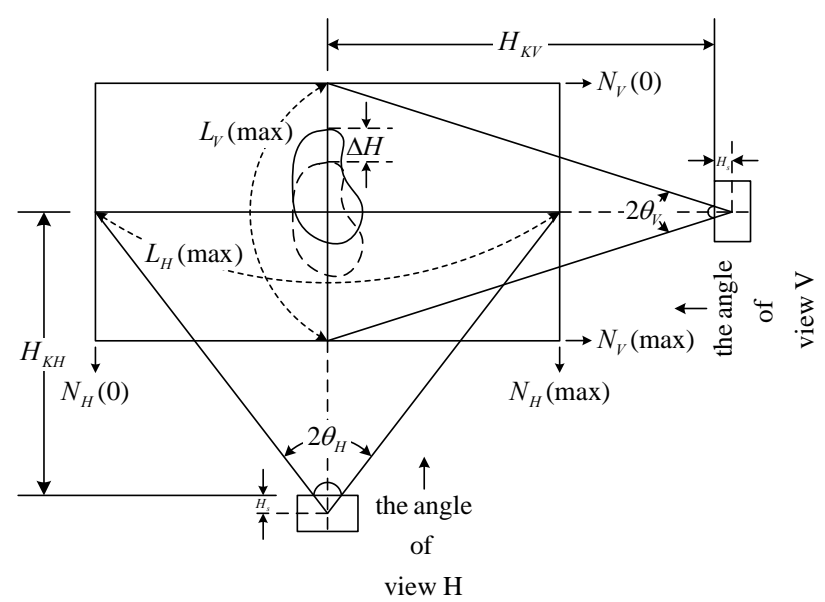

Fig. 3 Distance measurement via the proposed approach. 
Fig. 3 illustrates the image from the horizontal (view $\mathrm{H}$ ) and vertical (view $\mathrm{V}$ ) view angles respectively for a single image. Note that a single CCD camera, rather than two CCD cameras, is used at the same. The movement of the CCD camera is $\Delta h$, resulting a pixel variation of $\Delta N$ in the image. To better explain the proposed approach, the observation of image in Fig. 3 from the vertical angle is re-drawn as Fig. 4, where the relationship between the pixel variation $\Delta N$, camera movement $\Delta h$, view angle $\theta_{v}$, and distance from the targeted object $H_{K}$ can be better perceived.

\section{Three-Dimensional Measurement via the proposed approach}

While the photographing distance is $H_{K}$, the height of triangle $\left(P_{O}, P_{L}, P_{R}\right)$ will be $H_{K}$. The distance between $P_{L}$ and $P_{R}$ is the maximal real-world distance covered by the vertical view angle $2 \theta_{V}$. Previous research [7]-[9] has already shown that the scan time between two points in the image is proportional to their corresponding distance. As far as CCD camera is concerned, pixel counts and distance between $P_{L}$ and $P_{R}$ are in direct proportion. Based on the relationship among the pixel variation $\Delta N$ and camera movement $\Delta h, L_{V}(\max )$ can be expressed as follows:

$$
L_{V}(\max )=\frac{\Delta h}{\Delta N} \times N_{V}(\max )
$$

, where $N_{V}(\max )$ is the maximal pixels in a vertical scan line of an image frame. By a triangular relationship, when the included angle and the base line are known, the height $\left(H_{K V}\right)$ can be obtained As:

$$
H_{K V}=\frac{1}{2} L_{V}(\max ) \times \cot \theta_{V}-H_{S}
$$

, where $H_{S}$ is the distance between CCD camera and optic origin, and $2 \theta_{v}$ is vertical view angle of the CCD camera. $H_{S}$ and $\theta_{V}$ are the intrinsic parameters of measurement. How $H_{S}$ and $\theta_{V}$ can be accurately derived for various kinds of CCD cameras will be fully elaborated in next section so as to achieve a better measuring accuracy. As shown in Fig $4, L_{A}$ and $L_{B}$ are height of the targeted object under measurement, thus $L_{A}=L_{B}, N_{A}=N_{B}$. The formula for the height of the targeted object can be expressed as:

$$
L_{A}=L_{B}=\frac{N_{A}}{\Delta N} \times \Delta h=\frac{N_{B}}{\Delta N} \times \Delta h
$$

No matter the observations are made either from the horizontal or vertical view angle, the photographing distance between the CCD camera and the targeted object remains the same, i.e. $H_{K V}=H_{K H}$. The formula of the maximal real-world distance $L_{H}$ (max) when viewing from the horizontal direction can be expressed as:

$$
L_{H}(\max )=2\left(H_{K V}+H_{S}\right) \times \tan \theta_{H}
$$

Assume that the distance between two points $C_{1}$ and $C_{2}$ on the targeted object is $L_{C}$. Based on the pixel numbers between points $C_{1}$ and $C_{2}, L_{C}$ can be obtained via the formula below:

$$
L_{C}=\frac{N_{C}}{N_{H}(\max )} \times L_{H}(\max )
$$

, where $N_{H}(\max )$ is the maximal pixel numbers in the horizontal scan line of the CCD camera and $2 \theta_{H}$ is horizontal view angle. As results, (2) stands for the photographing distance between the CCD camera and the targeted object ( $\mathrm{Z}$ direction). (3) and (5) represent the height (Y direction) and width ( $\mathrm{X}$ direction) of the targeted object, respectively. By doing so, three dimensional measurement of a distant object can be obtained by using only a single CCD camera via the proposed measuring method.

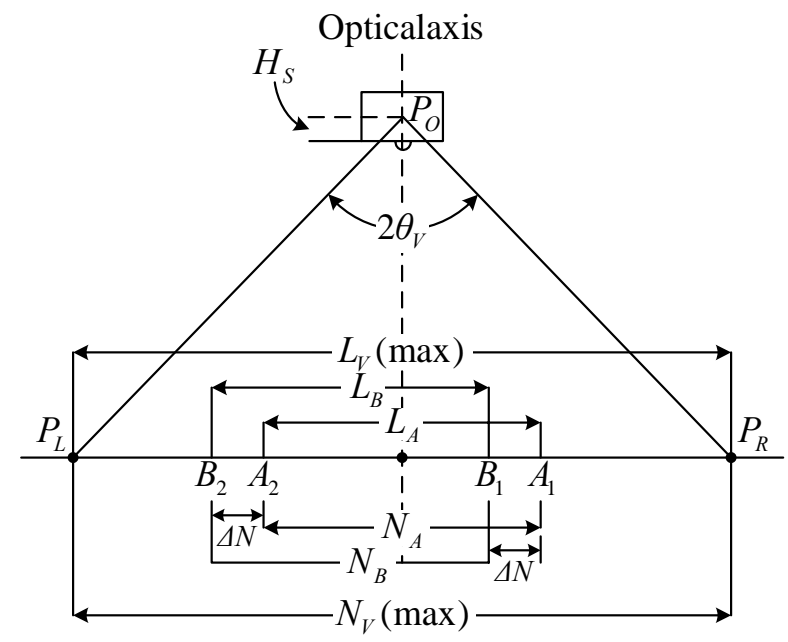

Fig. 4 Illustration of measurement from the V view angle. 


\section{Determination of the intrinsic parameters for $C C D$ cameras}

A set of intrinsic parameters of the CCD camera are required for using the proposed distance measuring system, including $H_{S}, \theta_{V}, \theta_{H}$. To improve measuring accuracy, a proposed measuring scheme is proposed in this section for identifying these parameters.

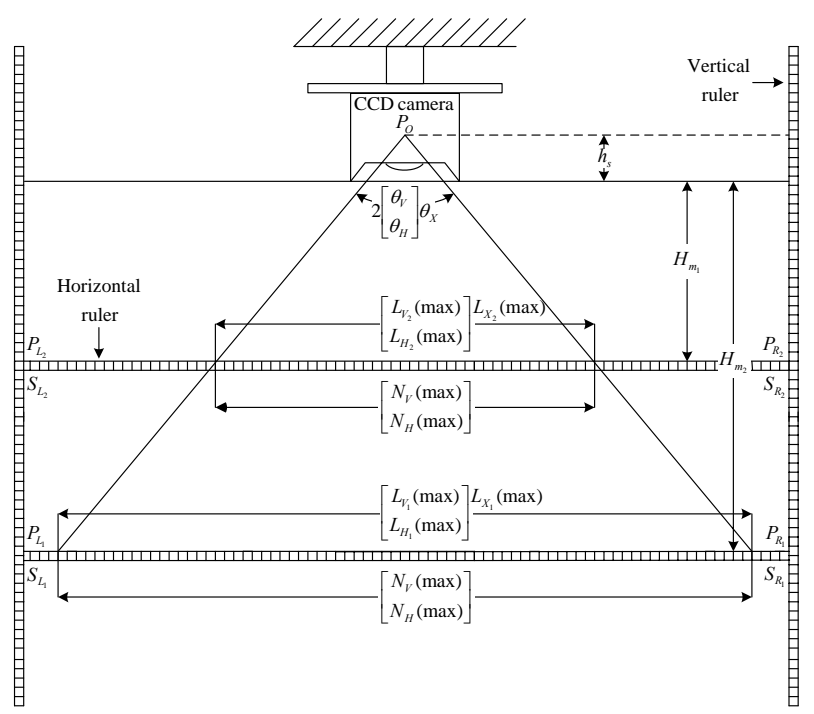

Fig. 5 Mechanism for obtaining intrinsic parameters for a CCD camera

As shown in Fig. 5, when the horizontal scan line of the CCD camera is parallel to the horizontal ruler, we have $\theta_{X}=\theta_{H} \quad, \quad L_{X_{1}}(\max )=L_{H_{1}}(\max )$ and, $L_{X 2}(\max )=L_{X_{2}}(\max )$, indicating the measuring parameters are established from the horizontal view angle. Turning the CCD camera 90 degrees, the measuring parameters are then established from the vertical view angle, i.e. $\theta_{X}=\theta_{V}, L_{X_{1}}(\max )=L_{V_{1}}(\max )$, $L_{X_{2}}(\max )=L_{V_{2}}(\max )$.

When the horizontal ruler is positioned at $S_{L_{1}}$ and $S_{R_{1}}$, the height will be $H_{m_{1}}$ and distance between points $P_{L_{1}}$ and $P_{R_{1}}$ on the image is $L_{X_{1}}$ (max). When the horizontal ruler is positioned at $S_{L 2}$ and $S_{R 2}$, the height will be $H_{m_{2}}$ and distance between points $P_{L_{2}}$ to $P_{R_{2}}$ on the image will be $L_{X_{2}}$ (max). As a result of the triangle theorem by observing triangles $\Delta\left(P_{O}, P_{L_{1}}, P_{K_{1}}\right)$ and $\Delta\left(P_{O}, P_{L_{2}}, P_{K_{2}}\right)$, we have the following relationship:

$$
H_{S}=\frac{H_{m_{1}} \times L_{X_{2}}(\max )-H_{m_{2}} \times L_{X_{1}}(\max )}{L_{X_{1}}(\max )-L_{X_{2}}(\max )}
$$

, where $X=V$ or $H$

$$
\cot \theta_{X}=\frac{2\left(H_{m_{1}}-H_{m_{2}}\right)}{L_{X_{1}}(\max )-L_{X_{2}}(\max )}
$$

, where $X=V$ or $H$

In an attempt to validate the performance of the proposed approach, a Panasonic DMC-LX1 CCD camera is used in this paper for all the experiments conducted. The measuring parameters associated with the CCD camera include $H_{S}=0.4 \mathrm{~cm}, \cot \theta_{V}=2.82$, and $\cot \theta_{H}=2.11$. The horizontal and vertical resolution in terms of pixels of the CCD camera are $N_{H}(\max )=3264$ and $N_{V}(\max )=2448$, respectively. When these measuring parameters are available, the height and the width of the targeted object in addition to the photographing distance can be obtained via the proposed method. Without making changes to the structure of the CCD camera, the proposed measuring system can easily achieve the objective of three-dimensional measurement of a targeted object by moving the camera straight up or down.

\section{Experiment results}

As shown in Fig. 1, the proposed measuring system moves the CCD camera with a specific distance in the vertical direction for obtaining the measurement of a targeted object. Measurement results and photos during the experiments are presented below.

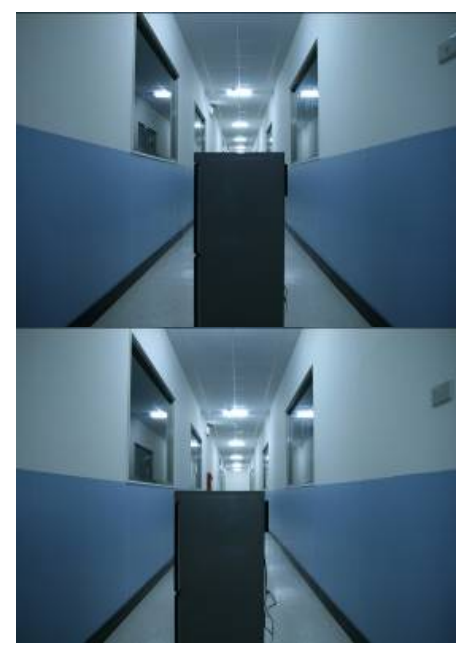

Fig.6 Photos before and after camera movement at photographing distance $H_{K}=140 \mathrm{~cm}$ 


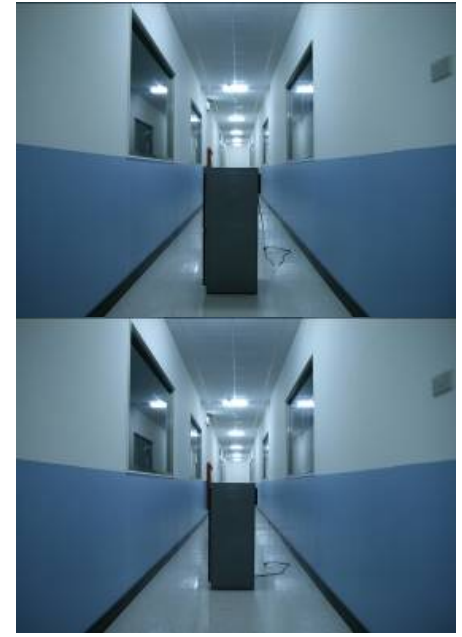

Fig. 7 Photos showing different photographing distances with $\Delta h=10 \mathrm{~cm}$

As demonstrated in the experiment results, the measurement accuracy via the proposed system has been significantly improved in comparison to the IBDMS (Image-Based Distance Measuring System) [7]-[9].

\section{Conclusion}

In this paper, we have proposed a measuring system based on the movement of a CCD camera along the vertical direction. Neither extra distance-measuring sensors nor physical measuring rulers are required to achieve a satisfactory measurement via the proposed method. As long as the camera movement becomes available, we can determine the pixel counts for a targeted object due to the camera movement to calculate the photographing distance as well as the height and width of the object. Because of the proposed method to accurately determine the intrinsic parameters for various CCD cameras, any camera can therefore be adopted to obtain satisfactory results of three-dimensional measurement via the proposed method. As demonstrated in the experiment results, distance information can be easily incorporated into the photos taken. On the basis of the existing research results, the next objective of the research team is to investigate as to how automatic distance measurement can be achieved based on only one vertical scan line.

\section{Reference:}

[1] Alessio Carullo, Franco Ferraris, and Salvatore Graziani, "Ultrasonic Distance Sensor Improvement Using a Two-Level Neural Network”, IEEE Transactions on Instrumentation and Measurement, Vol. 45, No. 2, pp.677-682, April 1996.
[2] A.Caarullo and M. Parvis, "An ultrasonic sensor for distance measurement in automotive applications," IEEE Sensors Journal, Vol. 1, No. 2, pp.143-147, 2001.

[3] K. Osugi, K. Miyauchi, N. Furui, and H. Miyakoshi, "Development of the scanning laser radar for ACC system," JSAE Rev. 20, pp.579-554, 1999.

[4] H.-T. shin, "Vehicles Crashproof Laser Radar," M.S. thesis, Opt. Sci. Center, National Central Unive., Chung Li City, Taiwan, R.O.C., 2000.

[5] Kanade, T., Kano, H., Kimuram S., "Development of a Video-Rate Stereo Machine”, Proc 1995 IEEE/RSJ Int. Conf. on Intelligent Robots and Systems, Pittsburgh, USA, August, 1995, Vol. 3, pp.95-100(1995).

[6] Tanaka, Y, gofuku, A., Nagai, I., Mohamed, A.:Development of a Compact Video-rate Range finder and its application, Proc. 3rd Int. Conf. on Advanced Mechatronics, Okayama, Japan, August, 1998, pp.97-102.

[7] Ming-Chih Lu, Wei-Yen Wang, and Hung-Hsun Lian, "Image-Based hight measuring system for liquid or particles in tanks," IEEE International Conference on Networking, Sensing and Control, Vol.1, pp.24-29, 2004.

[8] Ming-Chih Lu, Wei-Yen Wang, and Chun-Yen chu, "Optical-Based Distance Measuring System (ODMS)," The Eighth International Conference On Automation Technology, pp.282-285, 2005.

[9] Ming-Chih Lu, Wei-Yen Wang, and Chun-Yen Chu, "Image-Based Distance and Area Measuring System,” IEEE Sensors Journal. Vol. 6, No.2, pp.495-503, April 2006.

[10] M. A. Sid-Ahmed and M. T. Boraie, "Dual camera calibration for 3-D machine vision metrology," IEEE Trans. Instrum. Meas., vol. 39, no. 3, pp.512-516, Jun. 1990.

[11] C. Liguori, A. Pietrosanto, and A. Paolillo, “An on-line stereo vision system for dimensional measurements on rubber extrusions," in Proc. 11th IMEKO TC4 Int. Sym., Lisbon, Portugal, Sep 13/14, 2001, pp.15-19.

[12] J. Weng,P. Cohen, and M. Herniou, "Camera calibration with distortion models and accuracy evaluation," IEEE Trans. Pattern Anal. Mach. Intell., vol. 14, no. 10, pp.965-980, Oct.1992.

[13] M. Rebiai, S. N Mansouri, F. Pinson, and B. B. Tichit, "Image distortion form zoom lenses: Modeling and digital correction," in Proc. Int. Broad-casting Convention, 1992, pp.438-411. 
(A) Measurement of photographing distances:

Table 1 Results of distance measurement at $\Delta h=10 \mathrm{~cm}$

\begin{tabular}{|c|c|c|c|c|c|c|}
\hline $\begin{array}{c}\text { Actual } \\
\text { distance }\left(H_{K}\right) \\
(\mathrm{cm})\end{array}$ & 140 & 180 & 220 & 300 & 400 & 500 \\
\hline $\begin{array}{c}\text { Measured } \\
\text { distance }\left(H_{K}{ }^{*}\right) \\
(\mathrm{cm})\end{array}$ & 139.912 & 180.553 & 220.508 & 301.057 & 400.958 & 501.663 \\
\hline Error & $-0.063 \%$ & $0.307 \%$ & $0.231 \%$ & $0.352 \%$ & $0.240 \%$ & $0.333 \%$ \\
\hline
\end{tabular}

(B) Measurement of height and width:

Table 2 Results of height measurement at $\Delta h=10 \mathrm{~cm}$

\begin{tabular}{|c|c|c|c|c|c|c|}
\hline $\begin{array}{c}\text { Actual } \\
\text { distance }\left(H_{K}\right) \\
(\mathrm{cm})\end{array}$ & 140 & 180 & 220 & 300 & 400 & 500 \\
\hline $\begin{array}{c}\text { Actual } \\
\text { height }\left(L_{A}\right) \\
(\mathrm{cm})\end{array}$ & 50 & 50 & 50 & 50 & 50 & 50 \\
\hline $\begin{array}{c}\text { Measured } \\
\text { height }\left(L_{A}{ }^{*}\right) \\
(\mathrm{cm})\end{array}$ & 49.647 & 50.092 & 50.144 & 50.262 & 49.942 & 49.818 \\
\hline Error & $-0.706 \%$ & $0.183 \%$ & $0.288 \%$ & $0.524 \%$ & $-0.116 \%$ & $-0.364 \%$ \\
\hline
\end{tabular}

Table 3 Results of width measurement at $\Delta h=10 \mathrm{~cm}$

\begin{tabular}{|c|c|c|c|c|c|c|}
\hline $\begin{array}{c}\text { Actual } \\
\text { distance }\left(H_{K}\right) \\
(\mathrm{cm})\end{array}$ & 140 & 180 & 220 & 300 & 400 & 500 \\
\hline $\begin{array}{c}\text { Actual } \\
\text { height }\left(L_{A}\right) \\
(\mathrm{cm})\end{array}$ & 40 & 40 & 40 & 40 & 40 & 40 \\
\hline $\begin{array}{c}\text { Measured } \\
\text { height }\left(L_{A}{ }^{*}\right) \\
(\mathrm{cm})\end{array}$ & 40.365 & 40.234 & 40.227 & 40.172 & 40.203 & 40.144 \\
\hline Error & $0.911 \%$ & $0.585 \%$ & $0.567 \%$ & $0.431 \%$ & $0.506 \%$ & $0.359 \%$ \\
\hline
\end{tabular}

